\title{
A novel therapeutic target for triple negative breast cancer
}

\author{
Raj Kumar* \\ Department of Basic Sciences, The Commonwealth Medical College, Scranton, USA
}

Breast cancer is the fifth most common cause of cancer death, and the leading cause of cancer death in women. Breast cancer is a complex disease entity with different biological characteristics and clinical behavior. The heterogeneity of breast cancers makes them both a fascinating and challenging solid tumor to diagnose and treat. The treatment options currently available to patients diagnosed with breast cancer are surgery, radiation therapy, chemotherapy, and hormone therapy with an aim to eliminate the cancer, avoid the production of metastases, and prevent future remission. Gene expression profiling has yielded expression signatures from which prognostic tests can be derived to facilitate clinical decision making in breast cancer patients. The use of cytotoxic chemotherapy in both advanced and early stage breast cancer has made significant progress in the last decade or so with several landmark studies identifying clear survival benefits for newer therapies [1].

There are four clinically relevant breast cancer phenotypes currently recognized: luminal A (estrogen receptor $\left[\mathrm{ER}^{+}\right]$, progesterone receptor $\left[\mathrm{PR}^{+}\right]$, human epidermal growth factor receptor 2 [HER2- ${ }^{-}$, Ki67 low), luminal $\mathrm{B}\left(\mathrm{ER}^{+}, \mathrm{HER}^{-}, \mathrm{PR}^{-}\right.$, or Ki67 high), triple-negative breast cancer (TNBC; $\mathrm{ER}^{-}, \mathrm{PR}^{-}$, and HER2-), and HER2 over-expressing tumors $\left(\right.$ HER2 $2^{+}$). TNBC accounts for $10-20 \%$ of all invasive breast cancers and is frequently diagnosed in younger and premenopausal women and is highly prevalent in African American women. Since TNBCs test negative for ER, PR, and HER2, the cancer is unlikely to respond to hormone-and/or HRE2-based therapies. Hence, surgery and chemotherapy, individually or in combination are the only available modalities. Optimal conditions for the therapeutic assessment of women with TNBC and for the management of their disease have yet to be validated in prospective investigations. Several studies have shown that TNBC is highly prone to metastasis and recurrence. TNBC tumors relapse more frequently in spite of good initial response to chemotherapy, and have a worse prognosis than hormone receptorpositive, luminal subtypes. Finding effective treatment targets for TNBC has proven difficult so far. TNBCs are reported to respond to neo-adjuvant chemotherapy, but overall, survival in patients with such tumors is still poor [2]. Next-generation sequencing of TNBC has suggested that actionable mutations occur in only a small subset of these cancers and do not completely predict survival [3].

Given the poor outlook and lack of sustained response to conventional therapies, TNBC has been the subject of intense studies on new therapeutic approaches in recent years. The development of targeted cancer therapies, often in combination with established chemotherapy, has been applied to a number of new clinical studies in recent years. Therefore, there is an urgent need to develop new and novel therapeutic management of these patients that requires more aggressive intervention. Currently lot of research is going on to further characterize TNBC with different molecular markers and find targets for therapy in order to improve its outcome [4]. Biomarker(s) may be useful as prognostic or predictive indicators as well as suggest possible targets for novel therapies. Targeted therapy directed against many biomarkers has not shown significant improvement in outcome in TNBC. Therefore, the emphasis must be put on research for effective drug targets for the treatment of TNBC, which could be translated into clinical uses.

Glucocorticoids have been widely used as co-adjuvants in the treatment of solid tumors including breast cancer, but glucocorticoid treatment may be associated with poor therapeutic response or prognosis [5]. Glucocorticoid-mediated antagonism of paclitaxel was the first evidence suggesting that synthetic glucocorticoids and possibly high levels of secreted endogenous cortisol could inhibit the effectiveness of chemotherapy treatment by blocking tumor cell death [6]. It has been reported that the higher glucocorticoid receptor (GR) expression and activation initiates potent anti-apoptotic signaling pathways in breast epithelial cells, at least in part, via transcriptional regulation of genes encoding cell survival pathway [7]. For example, genes encoding the anti-apoptotic proteins, serum and glucocorticoid inducible protein kinase-1 (SGK1) and mitogen-activated protein kinase phosphatase-1 (MKP1/DUSP1), are up-regulated following GR activation [8]. Most TNBC cell lines express GR, and higher GRexpressing tumors have a significantly worse long-term prognosis [9].

A study showed that GR antagonists could potentiate chemotherapy-induced cytotoxicity in TNBC by blocking GRmediated tumor cell survival via inhibiting associated gene expression that are usually activated by endogenous glucocorticoids, thereby augmenting chemotherapy-induced cell death and decreasing in vivo TNBC tumor growth [10]. Another study reported severely reduced cell viability and proliferation of TNBC cells treated with a peptide that contains the LXXLL-motif of the human steroid receptor coactivators-1 (SRC-1), a member of p160 group of proteins [11]. Extensive studies have shown that the LXXLL sequence is necessary and sufficient for the binding of p160 proteins to steroid hormone receptors including GR and for stimulation of transcriptional activity. A strong correlation between SRC-1 expression and the pro-survival progression of breast cancer is well established, supporting its potential as a target for specific therapeutic intervention in the clinical management of TNBC.

In a recent study, the treatment of TNBC cells with Hsp90 inhibitors resulted in GR degradation and decreased GR-mediated gene expression [12]. This GR degradation also sensitized TNBC

Correspondence to: Raj Kumar, Ph.D., Professor and Director of Research, Department of Basic Sciences, The Commonwealth Medical College, 525 Pine Street, Scranton, PA-18509, USA, Tel: 570-504-9675, Fax: 570-504-9660, E-mail: rkumar@tcmc.edu

Received: April 10, 2016; Accepted: April 20, 2016; Published: April 22, 2016 
cells to paclitaxel-induced cell death both in vitro and in vivo [12]. These findings suggest that GR-regulated anti-apoptotic and proproliferative signaling networks in TNBC may be disrupted by $\mathrm{Hsp} 90$ inhibitors. However, the efficacy in inhibiting TNBC cell growth through these GR antagonistic means has been limited. Therefore, efforts must be made to better understand the molecular mechanisms regulating the GR-mediated cell proliferation of TNBC cells with an aim of developing a GR-targeted therapeutics to treat this deadly disease. Future investigations should aim to maximize the integration of factors so that the relative impacts of multiple factors can be assessed both independently and in combination. Furthermore, molecular and genetic markers may help to determine potentially relevant subgroups likely to experience maximal or minimal beneficial impact.

\section{References}

1. Zeichner SB, Terawaki H, Gogineni K (2016) A Review of Systemic Treatment in Metastatic Triple-Negative Breast Cancer. Breast Cancer (Auckl) 10: 25-36. [Crossref]

2. Groheux D, Biard L, Giacchetti S, Teixeira L, Hindié E, et al. (2016) 18F-FDG PET/ CT for the Early Evaluation of Response to Neoadjuvant Treatment in Triple-Negative Breast Cancer: Influence of the Chemotherapy Regimen. J Nucl Med 57: 536-543. [Crossref]

3. Brenner DR, Brockton NT, Kotsopoulos J, Cotterchio M, Boucher BA, et al. (2016) Breast cancer survival among young women: a review of the role of modifiable lifestyle factors. Cancer Causes Control 27: 459-472. [Crossref]

4. García-Teijido P, Cabal ML, Fernández IP, Pérez YF (2016) Tumor-Infiltrating
Lymphocytes in Triple Negative Breast Cancer: The Future of Immune Targeting. Clin Med Insights Oncol 10: 31-39. [Crossref]

5. Orqueda AJ, Dansey MV, Español A, Veleiro AS, Bal de Kier Joffé E, et al. (2014) The rigid steroid 21-hydroxy-6,19-epoxyprogesterone (21OH-6,19OP) is a dissociated glucocorticoid receptor modulator potentially useful as a novel coadjuvant in breast cancer chemotherapy. Biochem Pharmacol 89: 526-535. [Crossref]

6. Herr I, Ucur E, Herzer K, Okouoyo S, Ridder R, et al. (2003) Glucocorticoid cotreatment induces apoptosis resistance toward cancer therapy in carcinomas. Cancer Res 63: 3112-3120. [Crossref]

7. Wu W, Chaudhuri S, Brickley DR, Pang D, Karrison T, et al. (2004) Microarray analysis reveals glucocorticoid-regulated survival genes that are associated with inhibition of apoptosis in breast epithelial cells. Cancer Res 64: 1757-1764. [Crossref]

8. Chen Z, Lan X, Wu D, Sunkel B, Ye Z, et al. (2015) Ligand-dependent genomic function of glucocorticoid receptor in triple-negative breast cancer. Nature Commun 6: 8323 .

9. Pan D, Kocherginsky M, Conzen SD (2011) Activation of the glucocorticoid receptor is associated with poor prognosis in estrogen receptor-negative breast cancer. Cancer Res71: 6360-6370.[Crossref]

10. Skor MN, Wonder EL, Kocherginsky M, Goyal A, Hall BA, et al. (2013) Glucocorticoid receptor antagonism as a novel therapy for triple-negative breast cancer. Clin Cancer Res 19: 6163-6172. [Crossref]

11. Tints K, Prink M, Neuman T, Palm K (2014) LXXLL Peptide converts transportan 10 to a potent inducer of apoptosis in breast cancer cells. Int J Mol Sci 15: 5680-5698. [Crossref]

12. Agyeman AS, Jun WJ, Proia DA, Kim CR, Skor MN, et al. (2016) Hsp90 inhibition results in glucocorticoid receptor degradation in association with increased sensitivity to paclitaxel in triple-negative breast cancer. Horm Cancer 7: 114-126. [Crossref]

Copyright: $(02016$ Kumar R. This is an open-access article distributed under the terms of the Creative Commons Attribution License, which permits unrestricted use, distribution, and reproduction in any medium, provided the original author and source are credited. 